\title{
Exact solution of the average run length for the cumulative sum chart for a moving average process of order $q$
}

\author{
Kanita Petcharat, Saowanit Sukparungsee, Yupaporn Areepong* \\ Department of Applied Statistics, Faculty of Applied Science, \\ King Mongkut's University of Technology North Bangkok, Bangkok 10800 Thailand
}

*Corresponding author, e-mail: yupaporna@kmutnb.ac.th

Received 13 Apr 2013

Accepted 27 Nov 2014

\begin{abstract}
In this paper we use a Fredholm integral equation approach to derive an explicit formula for the average run length (ARL) of a cumulative sum (CUSUM) chart for random observations described by a moving average process of order $q(\mathrm{MA}(q))$ with exponential white noise. We compare the computational times required for calculating the ARL from our exact formula with the computational times required for solving the Fredholm integral equations using a Gauss-Legendre numerical scheme. We find that the computational times are approximately $1 \mathrm{~s}$ for the explicit formula and approximately $13 \mathrm{~min}$ for the numerical integration scheme.
\end{abstract}

KEYWORDS: moving average process of order $q$, white noise, exponential distribution

MSC2010: 97N40 34K07 46N20

\section{INTRODUCTION}

The cumulative sum (CUSUM) chart is a common and effective graphical procedure for monitoring quality control in a manufacturing industry. The CUSUM chart ${ }^{1}$ is good for detecting small changes in observed parameters in statistical process control. CUSUM charts have been applied in a range of different areas. A review of CUSUM charts has been given by Mazalov and Zhuravlev ${ }^{2}$, who implemented CUSUM charts to identify change points in traffic in computer networks. Dong ${ }^{3}$ has employed CUSUM charts in economics and finance to detect turning points in IBM stock prices. Corbett and $\operatorname{Pan}^{4}$ have used CUSUM charts in environmental science to monitor emission data. Kennedy ${ }^{5}$ has applied CUSUM charts in queueing processes to compute the distribution of the first passage times for an $\mathrm{M} / \mathrm{M} / \mathrm{l}$ queue. CUSUM charts have also been used to calculate stopping times associated with sequential cumulative sum tests in health care and public health ${ }^{6,7}$.

A common characteristic of control charts is the average run length (ARL), which is defined as the expectation of the alarm time taken to trigger a signal about a possible change in parameters of a distribution. Ideally, an acceptable ARL for an incontrol process should be large enough to avoid an excessive number of false alarms. In this paper we adopt the notation for the in-control ARL as $\mathrm{ARL}_{0}=$ $\mathbb{E}_{\infty}(\tau)$ for the expectation of stopping time $\tau$ corresponding to a target value $T$ which is assumed to be large enough. The out-of-control ARL is denoted by $A R L_{1}$ and is defined as the expectation of delay time for a true alarm. This time should minimize the quantity

$$
\mathrm{ARL}_{1}=\mathbb{E}_{\theta}(\tau-\theta+1 \mid \tau \geqslant \theta)
$$

where $\mathbb{E}_{\theta}$ is the expectation under the assumption that a change-point occurs at a given time $\theta$.

In the literature, several methods have been described for evaluating the ARL of CUSUM and EWMA procedures, e.g., Monte Carlo simulation (MC), integral equation (IE) ${ }^{8,9}$, and Markov chain approximation ${ }^{10,11}$. Zhonghua et al ${ }^{12}$ intensively reviewed the integral equation and Markov chain methods for computing the average run length. Sukparungsee and Novikov ${ }^{13}$ derived closed-form formulae for the ARL for light-tailed distributions using a martingale approach. Areepong ${ }^{14}$ presented an analytical derivation of the ARL of an EWMA chart for exponentially distributed observations using an integral equation approach. Mititelu et $\mathrm{al}^{15}$ used the Fredholm integral equations approach to derive analytical expressions for the ARL of EWMA 
and CUSUM charts when observations have a hyperexponential distribution. Petcharat et al ${ }^{16}$ derived closed-form expressions for the ARL of CUSUM charts for Pareto and Weibull distributed observations by approximating these distributions with the hyperexponential distribution.

CUSUM control charts have traditionally been designed for independent and identically distributed (i.i.d.) observations. However, in real life problems, correlated observations may be present in some processes ${ }^{17}$ and these correlations can affect properties of CUSUM charts. Jacob and Lewis ${ }^{18}$ analysed autoregressive-moving average processes of order $(1,1)(\operatorname{ARMA}(1,1))$ when observations are exponentially distributed with exponential white noise. Lawrance and Lewis ${ }^{19}$ studied exponential moving average processes of the first order. These processes are important in queueing and network problems. Mohamed and Hocine ${ }^{20}$ used Bayesian methods to analyse an autoregressive model with exponential white noise.

In this paper, we derive an analytical expression for the ARL of a CUSUM chart when the random observations are modelled as a moving average process of order $q(\mathrm{MA}(q))$ with exponential white noise. We then use the Banach fixed point theorem (see, e.g., Ref. 21) to prove the existence and uniqueness of solutions of this analytical expression. Finally, we compare numerical results obtained from the analytical expression for the ARL of MA $(q)$ processes with results obtained from a numerical solution of an integral equation for the ARL. We also compare CPU times for the analytical and integral equation computations.

\section{AVERAGE RUN LENGTH FOR CUSUM CHARTS FOR MOVING AVERAGE PROCESSES OF ORDER $q$ WITH EXPONENTIAL WHITE NOISE}

A CUSUM chart is most often implemented for monitoring and detecting small changes in parameters of a given distribution. Let $\xi_{n}$ be the observations of a stationary moving average process of order $q$ with exponential white noise defined as $Z_{n}=\xi_{n}$ $\theta_{1} \xi_{n-1}-\theta_{2} \xi_{n-2}-\ldots-\theta_{q} \xi_{n-q}$, where $\left|\theta_{i}\right|<1$, for $i=$ $1,2, \ldots$, and $\xi_{n} \sim \operatorname{Exp}(\lambda)$. The CUSUM recurrence chart is defined by

$$
X_{n}=\max \left(X_{n-1}+Z_{n}-a, 0\right), \quad n=1,2, \ldots,
$$

where $X_{n}$ are random variables, and $a$ is a nonzero CUSUM reference value. The corresponding stopping time for (1) is defined as

$$
\tau_{b}=\inf \left\{n>0: X_{n}>b\right\}
$$

where $b$ denotes the out-of-control parameter limit. Let $\mathbb{P}_{x}$ and $\mathbb{E}_{x}$ be the probability measure and the induced expectation corresponding to the initial value $X_{0}=x$. Then the ARL $=j(x)=\mathbb{E}_{x}\left(\tau_{b}\right)<\infty$ is the unique solution of the ARL integral equation ${ }^{15}$

$$
\begin{aligned}
j(x)=1+\mathbb{E}_{x}\left[I\left\{0<X_{1}<b\right\} j\left(X_{1}\right)\right] & \\
+ & \mathbb{P}_{x}\left\{X_{1}=0\right\} j(0), \quad x<b
\end{aligned}
$$

where the indicator function $I\left(0<X_{1}<b\right)=1$ if $0<X_{1}<b$ and 0 otherwise.

\section{Uniqueness of solution of an integral equation for the ARL}

Mititelu et al ${ }^{15}$ have used the integral equation approach to analyse the ARL for first order stationary autoregressive processes with exponential white noise for the case of i.i.d. random variables. In this section, we use the integral equation approach to prove the uniqueness of solutions for the ARL for stationary moving average processes by using the following theorem.

Theorem 1 (Banach Fixed Point Theorem) . Let $(X, d)$ be a non-empty complete metric space with a contraction mapping $T: X \rightarrow X$. Then $T$ admits a unique fixed-point $x^{*} \in X$ (i.e., $T\left(x^{*}\right)=x^{*}$ ). Furthermore, $x^{*}$ can be found as follows: start with an arbitrary element $x_{0} \in X$ and define a sequence $\left\{x_{n}\right\}$ by $x_{n}=T\left(x_{n-1}\right)$, then $x_{n} \rightarrow x^{*}$.

A stationary first order moving average process, MA(1), with exponential white noise $\xi_{n}$ is defined by the recurrence relation $Z_{n}=\xi_{n}-\theta_{1} \xi_{n-1}$, where $\left|\theta_{1}\right|<1$, and $\xi_{n} \sim \operatorname{Exp}(\lambda)$. A stationary second order moving average process, MA(2) with exponential white noise $\xi_{n}$ is defined by the recurrence $Z_{n}=\xi_{n}-\theta_{1} \xi_{n-1}-\theta_{2} \xi_{n-2}$, where $\left|\theta_{1}+\theta_{2}\right|<1$, $\left|\theta_{i}\right|<1$, for $i=1,2$, and $\xi_{n} \sim \operatorname{Exp}(\lambda)$. Following the method used for deriving (3) for MA(1), we can derive an integral equation for an $\operatorname{MA}(q)$ process. We obtain

$$
\begin{aligned}
& j(x)=1+\lambda \mathrm{e}^{\lambda\left(x-a-\left(\theta_{1} \xi_{0}+\cdots+\theta_{q} \xi_{1-q}\right)\right)} \int_{0}^{b} j(y) \mathrm{e}^{-\lambda y} \mathrm{~d} y \\
& +\left(1-\mathrm{e}^{\lambda\left(x-a-\left(\theta_{1} \xi_{0}+\cdots+\theta_{q} \xi_{1-q}\right)\right)}\right) j(0), x \in[0, a) .
\end{aligned}
$$

Since the right-hand side of (4) is continuous, the solution of (4) is also a continuous function. Now, consider the non-empty complete metric space $\left(C(I),\|\|_{\infty}\right)$, where $C(I)$ denotes the space of all continuous functions on a compact interval $I$ and the norm $\|j\|_{\infty}=\sup _{x \in I}|j(x)|$. Recall that an 
operator $T$ is a contraction (see, e.g., Ref. 21) if there exists a real constant $0 \leqslant q<1$ such that $\left\|T\left(j_{1}\right)-T\left(j_{2}\right)\right\| \leqslant q\left\|j_{1}-j_{2}\right\|$ for all $j_{1}, j_{2} \in C(I)$. In our case, let $T$ be an operator in the class of all continuous functions $C(I)$, where $I=[0, a]$, and let $T$ be defined by

$$
\begin{aligned}
& T(j(x)) \\
& \quad=1+\lambda \mathrm{e}^{-\lambda\left(a-x+\theta_{1} \xi_{0}+\cdots+\theta_{q} \xi_{1-q}\right)} \int_{0}^{b} j(y) \mathrm{e}^{-\lambda y} \mathrm{~d} y \\
& +\left(1-\mathrm{e}^{-\lambda\left(a-x+\theta_{1} \xi_{0}+\cdots+\theta_{q} \xi_{1-q}\right)}\right) j(0), x \in[0, a) .
\end{aligned}
$$

Then (5) can be written in operator form as $T(j(x))=j(x)$. To prove the uniqueness of solution of (5) we first prove the following theorem.

Theorem 2 On the metric space $\left(C(I),\|\|_{\infty}\right)$ with the norm $\|j\|_{\infty}=\sup _{x \in I}|j(x)|$ the operator $T$ is a contraction.

Proof: To show that $T$ is a contraction we need to prove that for all $x \in I$ and $j_{1}, j_{2} \in C(I)$ we have the inequality $\left\|T\left(j_{1}\right)-T\left(j_{2}\right)\right\| \leqslant q\left\|j_{1}-j_{2}\right\|$, where $0 \leqslant q<1$. From (5) we obtain

$$
\begin{aligned}
& \| T\left(j_{1}\right)-T\left(j_{2}\right) \|_{\infty} \leqslant \sup _{x \in[0, a)}\{ \\
&\left|j_{1}(0)-j_{2}(0)\right|\left(1-\mathrm{e}^{-\lambda\left(a-x+\theta_{1} \xi_{0}+\cdots+\theta_{q} \xi_{1-q}\right)}\right) \\
&+\lambda \mathrm{e}^{-\lambda\left(a-x+\theta_{1} \xi_{0}+\cdots+\theta_{q} \xi_{1-q}\right)} \\
&\left.\quad \times \int_{0}^{b}\left[j_{1}(y)-j_{2}(y)\right] \mathrm{e}^{-\lambda y} \mathrm{~d} y\right\} \\
& \leqslant\left\|j_{1}-j_{2}\right\|_{\infty} \sup _{x \in[0, a)}\{ \\
& 1-\mathrm{e}^{-\lambda\left(a-x+\theta_{1} \xi_{0}+\cdots+\theta_{q} \xi_{1-q}\right)} \\
&\left.+\lambda \mathrm{e}^{-\lambda\left(a-x+\theta_{1} \xi_{0}+\cdots+\theta_{q} \xi_{1-q}\right)} \int_{0}^{b} \mathrm{e}^{-\lambda y} \mathrm{~d} y\right\} \\
&=\left\|j_{1}-j_{2}\right\|_{\infty} \\
& \sup _{x \in[0, a)}\left\{1-\mathrm{e}^{-\lambda\left(a-x+\theta_{1} \xi_{0}+\cdots+\theta_{q} \xi_{1-q}\right)-\lambda b}\right\} \\
&=\left(1-\mathrm{e}^{-\lambda\left(\theta_{1} \xi_{0}+\ldots+\theta_{q} \xi_{1-q}\right)-\lambda b}\right)\left\|j_{1}-j_{2}\right\|_{\infty} \\
&= q_{1}\left\|j_{1}-j_{2}\right\|_{\infty},
\end{aligned}
$$

where $0<q_{1}=\left(1-\mathrm{e}^{-\lambda\left(\theta_{1} \xi_{0}+\cdots+\theta_{q} \xi_{1-q}+b\right)}\right)<1$. We have used the triangle inequality for norms and the fact that $\left|j_{1}(0)-j_{2}(0)\right| \leqslant \sup _{x \in[0, a)}\left|j_{1}(x)-j_{2}(x)\right|=$ $\left\|j_{1}-j_{2}\right\|_{\infty}$.

Hence the uniqueness of the solution is guaranteed by Theorem 2 and the Banach Fixed Point Theorem.

\section{The exact solution for the ARL integral equation}

Next, we derive the explicit solution of the Fredholm integral equation (4).

Theorem 3 The solution of $T(j(x))=j(x)$ is

$$
\begin{array}{r}
j(x)=\mathrm{e}^{\lambda b}\left(1+\mathrm{e}^{\lambda\left(b+a+\theta_{1} \xi_{0}+\cdots+\theta_{q} \xi_{1-q}\right)}-\lambda b\right)-\mathrm{e}^{\lambda x}, \\
x \leqslant a .
\end{array}
$$

Proof: From (6), we have for $x \in[0, a)$ that

$$
\begin{aligned}
& j(x)=1+ \lambda \mathrm{e}^{\lambda\left(x-a-\theta_{1} \xi_{0}-\cdots-\theta_{q} \xi_{1-q}\right)} \int_{0}^{b} j(y) \mathrm{e}^{-\lambda y} \mathrm{~d} y \\
&+\left(1-\mathrm{e}^{-\lambda\left(a-x+\theta_{1} \xi_{0}+\ldots+\theta_{1} \xi_{1-q}\right)}\right) j(0) .
\end{aligned}
$$

Let $d=\int_{0}^{b} j(y) \mathrm{e}^{-\lambda y} \mathrm{~d} y$. The function $j(x)$ can then be written as

$$
\begin{aligned}
j(x)=1+ & \lambda \mathrm{e}^{\lambda\left(x-a-\theta_{1} \xi_{0}-\ldots-\theta_{q} \xi_{1-q}\right)} d \\
+ & \left(1-\mathrm{e}^{-\lambda\left(a-x+\theta_{1} \xi_{0}+\ldots+\theta_{q} \xi_{1-q}\right)}\right) j(0) .
\end{aligned}
$$

At $x=0$ we have

$$
\begin{aligned}
j(0)=1+\lambda \mathrm{e}^{\lambda\left(-a-\theta_{1} \xi_{0}-\ldots-\theta_{q} \xi_{1-q}\right)} d \\
+\left(1-\mathrm{e}^{-\lambda\left(a+\theta_{1} \xi_{0}+\ldots+\theta_{q} \xi_{1-q}\right)}\right) j(0) .
\end{aligned}
$$

Then from (9) we obtain

$$
j(0)=\mathrm{e}^{\lambda\left(a+\theta_{1} \xi_{0}+\ldots+\theta_{q} \xi_{1-q}\right)}+\lambda d .
$$

Substituting (10) into (8), we obtain

$$
\begin{aligned}
j(x)=1+\lambda & \mathrm{e}^{\lambda\left(x-a-\theta_{1} \xi_{0}-\cdots-\theta_{q} \xi_{1-q}\right)} d \\
+ & \left(1-\mathrm{e}^{-\lambda\left(a-x+\theta_{1} \xi_{0}+\cdots+\theta_{q} \xi_{1-q}\right)}\right) \\
& \times\left(\mathrm{e}^{\lambda\left(a+\theta_{1} \xi_{0}+\cdots+\theta_{q} \xi_{1-q}\right)}+\lambda d\right) \\
=1 & \mathrm{e}^{\lambda\left(a+\theta_{1} \xi_{0}+\cdots+\theta_{q} \xi_{1-q}\right)}+\lambda d-\mathrm{e}^{\lambda x} .
\end{aligned}
$$

Now, we can evaluate the constant $d$ from (11) as

$$
\begin{aligned}
d= & \int_{0}^{b} j(y) \mathrm{e}^{-\lambda y} \mathrm{~d} y \\
= & \int_{0}^{b}\left(1+\lambda d+\mathrm{e}^{\lambda\left(a+\theta_{1} \xi_{0}+\cdots+\theta_{q} \xi_{1-q}\right)}-\mathrm{e}^{\lambda y}\right) \\
& \quad \times \mathrm{e}^{-\lambda y} \mathrm{~d} y \\
= & \left(1+\lambda d+\mathrm{e}^{\lambda\left(a+\theta_{1} \xi_{0}+\cdots+\theta_{q} \xi_{1-q}\right)}\right) \int_{0}^{b} \mathrm{e}^{-\lambda y} \mathrm{~d} y \\
& -\int_{0}^{b} \mathrm{e}^{\lambda y-\lambda y} \mathrm{~d} y .
\end{aligned}
$$


Hence (12) can rewritten as

$$
\begin{array}{r}
d=\frac{\mathrm{e}^{\lambda b}}{\lambda}\left(1-\mathrm{e}^{-\lambda b}\right)\left(1+\mathrm{e}^{\lambda\left(a+\theta_{1} \xi_{0}+\cdots+\theta_{q} \xi_{1-q}\right)}\right) \\
-b \mathrm{e}^{\lambda b} .
\end{array}
$$

Finally, substituting the constant $d$ into (13), we obtain

$$
\begin{array}{r}
j(x)=\mathrm{e}^{\lambda b}\left(1+\mathrm{e}^{\lambda\left(a+\theta_{1} \xi_{0}+\cdots+\theta_{q} \xi_{1-q}\right)}-\lambda b\right)-\mathrm{e}^{\lambda x}, \\
x \geqslant 0 .
\end{array}
$$

\section{Numerical solution for the ARL integral equation}

In this section, we present a numerical method to compute the solution $j(x)=\mathbb{E}_{x}\left(\tau_{b}\right)$ of the integral equation (4) for the ARL of an MA(q) process with exponential white noise. We first rewrite (4) in the form

$$
\begin{aligned}
j(x) & =1+j(0) F\left(a-x+\theta_{1} \xi_{0}+\cdots+\theta_{q} \xi_{1-q}\right) \\
& +\int_{0}^{b} j(y) f\left(a-x+\theta_{1} \xi_{0}+\cdots+\theta_{q} \xi_{1-q}+y\right) \mathrm{d} y
\end{aligned}
$$

where $F(x)=1-\mathrm{e}^{-\lambda x}$ and $f(x)=(\mathrm{d} F(x) / \mathrm{d} x)=$ $\lambda \mathrm{e}^{-\lambda x}$.

Now, we can approximate the integral $j(x)$ using the Gauss-Legendre quadrature rule as follows:

$$
\begin{aligned}
& j\left(a_{i}\right) \approx 1+j(0) F\left(a-a_{i}+\theta_{1} \xi_{0}+\cdots+\theta_{q} \xi_{1-q}\right) \\
& \quad+\sum_{k=1}^{m} w_{k} j\left(a_{k}\right) f\left(a_{k}+a-a_{i}+\theta_{1} \xi_{0}+\cdots+\theta_{q} \xi_{1-q}\right),
\end{aligned}
$$

where $i=1,2, \ldots, m$, with the weights $w_{k}=$ $(b / m) \geqslant 0$ and $a_{k}=(b / m)\left(k-\frac{1}{2}\right)$ for $k=$ $1,2, \ldots, m$.

The integral equation (15) then becomes a system of $m$ linear equations (16) in the $m$ unknowns $j\left(a_{1}\right), j\left(a_{2}\right), \ldots, j\left(a_{m}\right)$. For numerical implementation, it is preferable to write the linear system (16) in a matrix form as follows. We write

$$
\left(\mathbf{I}_{m}-\mathbf{R}_{m \times m}\right) \mathbf{J}_{m \times 1}=\mathbf{B}_{m \times 1},
$$

where

$$
\mathbf{J}_{m \times 1}=\left(\begin{array}{c}
j\left(a_{1}\right) \\
j\left(a_{2}\right) \\
\vdots \\
j\left(a_{m}\right)
\end{array}\right)
$$

$$
\mathbf{B}_{m \times 1}=\left(\begin{array}{c}
1+j(0)\left(a-a_{1}+\theta_{1} \xi_{0}+\cdots+\theta_{q} \xi_{1-q}\right) \\
1+j(0)\left(a-a_{2}+\theta_{1} \xi_{0}+\cdots+\theta_{q} \xi_{1-q}\right) \\
\vdots \\
1+j(0)\left(a-a_{m}+\theta_{1} \xi_{0}+\cdots+\theta_{q} \xi_{1-q}\right)
\end{array}\right),
$$

and

$$
\mathbf{R}_{m \times m}=\left(\begin{array}{cccc}
r_{11} & r_{12} & \cdots & r_{1 m} \\
r_{21} & r_{22} & \cdots & r_{2 m} \\
\vdots & \vdots & \ddots & \vdots \\
r_{m 1} & r_{m 2} & \cdots & r_{m m}
\end{array}\right)
$$

with

$$
\begin{array}{r}
r_{i j}=F\left(a-a_{i}+\sum_{k=1}^{q} \theta_{k} \xi_{1-k}\right) \\
+w_{j} f\left(a_{j}+a-a_{i}+\sum_{k=1}^{q} \theta_{k} \xi_{1-k}\right), \\
1 \leqslant i, \quad j \leqslant m,
\end{array}
$$

and $\mathbf{I}_{m}=\operatorname{diag}(1,1, \ldots, 1)$ is the identity matrix of order $m$. If the inverse $\left(\mathbf{I}_{m}-\mathbf{R}_{m \times m}\right)^{-1}$ exists, then the unique solution of (17) is

$$
\mathbf{J}_{m \times 1}=\left(\mathbf{I}_{m}-\mathbf{R}_{m \times m}\right)^{-1} \mathbf{B}_{m \times 1} .
$$

Then the approximate values of $j\left(a_{1}\right), j\left(a_{2}\right), \ldots, j\left(a_{m}\right)$ can be obtained from the solution (21) and we may approximate the function $j(x)$ by the function

$$
\begin{aligned}
& j^{\mathrm{IE}}(x)=1+j(0) F\left(a-x+\theta_{1} \xi_{0}+\cdots+\theta_{q} \xi_{1-q}\right) \\
& \quad+\sum_{k=1}^{m} w_{k} j\left(a_{k}\right) f\left(a_{k}+a-x+\theta_{1} \xi_{0}+\cdots+\theta_{q} \xi_{1-q}\right)
\end{aligned}
$$

where $w_{k}=b / m$ and $a_{k}=(b / m)\left(k-\frac{1}{2}\right), \quad k=$ $1,2, \ldots, m$.

\section{NUMERICAL RESULTS}

In this section, we present a comparison between the closed form expression given in Theorem 3 for $j(x)=$ ARL for the CUSUM chart when random observations are a moving average order $q$ process with exponential white noise and the approximate numerical solution for the ARL $j^{\mathrm{IE}}(x)$ given in (22). As a measure of accuracy of this comparison we define the relative error as

$$
\varepsilon_{r}=\frac{\left|j(x)-j^{\mathrm{IE}}(x)\right|}{j(x)} .
$$


Table 1 Comparison of $\mathrm{ARL}_{0}$ values for MA(1) process from explicit formula (exact) and numerical approximation (IE) for $\theta_{1}=0.23,0.53,0.83, a=3.5,4, b=$ $0.38,1.7,2$, and $m=500$.

\begin{tabular}{|c|c|c|c|c|c|c|}
\hline \multirow{2}{*}{$\theta$} & \multirow[t]{2}{*}{$b$} & \multirow{2}{*}{ ARL } & \multicolumn{2}{|c|}{$a=3.5$} & \multicolumn{2}{|c|}{$a=4$} \\
\hline & & & $x=0$ & $x=2$ & $x=0$ & $x=2$ \\
\hline \multirow[t]{12}{*}{0.23} & \multirow[t]{4}{*}{0.38} & Exact & 60.85 & 54.46 & 100.39 & 94.00 \\
\hline & & IE & 60.38 & 54.40 & 100.35 & 93.97 \\
\hline & & Times & (11.09) & (11.08) & (11.09) & (11.07) \\
\hline & & $100 \varepsilon_{r}$ & 0.78 & 0.12 & 0.04 & 0.04 \\
\hline & \multirow[t]{4}{*}{1.7} & Exact & 223.32 & 216.93 & 371.32 & 364.94 \\
\hline & & IE & 222.95 & 216.57 & 370.70 & 364.32 \\
\hline & & Times & (11.13) & (11.13) & (11.04) & (11.05) \\
\hline & & $100 \varepsilon_{r}$ & 0.17 & 0.17 & 0.17 & 0.17 \\
\hline & \multirow[t]{4}{*}{2.0} & Exact & 299.58 & 293.19 & 499.37 & 94.002 \\
\hline & & IE & 298.99 & 292.62 & 498.38 & 492.01 \\
\hline & & Times & (11.16) & (11.20) & (11.09) & (11.09) \\
\hline & & $100 \varepsilon_{r}$ & 0.19 & 0.19 & 0.20 & ? ? \\
\hline \multirow[t]{12}{*}{0.53} & \multirow[t]{4}{*}{0.38} & Exact & 82.18 & 75.79 & 135.55 & 129.16 \\
\hline & & IE & 82.15 & 75.76 & 135.5 & 129.11 \\
\hline & & Times & (11.34) & (11.31) & (11.14) & (11.25) \\
\hline & & $100 \varepsilon_{r}$ & 0.04 & 0.04 & 0.04 & 0.04 \\
\hline & \multirow[t]{4}{*}{1.7} & Exact & 303.14 & 296.75 & 502.92 & 496.54 \\
\hline & & IE & 302.63 & 296.25 & 502.07 & 495.7 \\
\hline & & Times & (11.28) & (11.28) & (11.29) & $(11.29)$ \\
\hline & & $100 \varepsilon_{r}$ & 0.17 & 0.17 & 0.17 & 0.17 \\
\hline & \multirow[t]{4}{*}{2.0} & Exact & 407.33 & 400.94 & 677.01 & 670.62 \\
\hline & & IE & 406.53 & 400.15 & 675.67 & 669.29 \\
\hline & & Times & (11.3) & (11.18) & (11.30) & $(11.29)$ \\
\hline & & $100 \varepsilon_{r}$ & 0.2 & 0.2 & 0.2 & 0.2 \\
\hline \multirow[t]{12}{*}{0.83} & \multirow[t]{4}{*}{0.38} & Exact & 110.96 & 104.57 & 183.0 & 176.61 \\
\hline & & IE & 110.92 & 104.53 & 182.93 & 176.55 \\
\hline & & Times & (11.12) & (11.35) & (10.88) & (11.12) \\
\hline & & $100 \varepsilon_{r}$ & 0.04 & 0.04 & 0.04 & 0.04 \\
\hline & \multirow[t]{4}{*}{1.7} & Exact & 410.88 & 404.49 & 680.57 & 674.17 \\
\hline & & IE & 410.19 & 403.82 & 679.42 & 673.04 \\
\hline & & Times & (11.40) & (11.45) & (11.16) & (11.17) \\
\hline & & $100 \varepsilon_{r}$ & 0.17 & 0.17 & 0.17 & 0.17 \\
\hline & \multirow[t]{4}{*}{2.0} & Exact & 552.77 & 546.28 & 916.80 & 910.413 \\
\hline & & IE & 551.68 & 545.3 & 914.98 & 908.61 \\
\hline & & Times & (11.37) & (11.36) & (11.23) & (11.11) \\
\hline & & $100 \varepsilon_{r}$ & 0.2 & 0.18 & 0.2 & 0.2 \\
\hline
\end{tabular}

We used (14) and (22) to evaluate the ARL for the first order moving average process (MA(1)) with exponential white noise and parameters $\theta=$ $0.23,0.53,0.83, a=3.5,4$ and $b=0.38,1.7,2$. The numerical values with the corresponding relative errors are shown in Table 1. Table 1 shows that for an MA(1) process with $\lambda=1$ there is excellent agreement between the values for $\mathrm{ARL}_{0}$ computed from the exact expression $j(x)$ and from the numer-
Table 2 Comparison of $\mathrm{ARL}_{0}$ values for MA(2) process from explicit formula (exact) and numerical approximation (IE) for $\theta_{1}=0.2, \theta_{2}=0.2,0.4,0.6, a=3,4, b=$ $1,1.5,2$, and $m=500$.

\begin{tabular}{|c|c|c|c|c|c|c|c|}
\hline \multirow{2}{*}{$\theta_{1}$} & \multirow{2}{*}{$\theta_{2}$} & \multirow[t]{2}{*}{$b$} & \multirow{2}{*}{ ARL } & \multicolumn{2}{|c|}{$a=3$} & \multicolumn{2}{|c|}{$a=4$} \\
\hline & & & & $x=0$ & $x=1$ & $x=0$ & $x=1$ \\
\hline \multirow{36}{*}{0.2} & \multirow{12}{*}{0.2} & \multirow{4}{*}{1.0} & Exact & 80.45 & 78.73 & 220.41 & 218.69 \\
\hline & & & IE & 80.37 & 78.66 & 220.19 & 218.47 \\
\hline & & & Times & (11.58) & (12.61) & (12.0) & (12.34) \\
\hline & & & $100 \varepsilon_{r}$ & 0.1 & 0.1 & 0.1 & 0.1 \\
\hline & & \multirow[t]{4}{*}{1.5} & Exact & 131.05 & 129.33 & 361.8 & 360.08 \\
\hline & & & IE & 130.86 & 129.14 & 361.26 & 359.55 \\
\hline & & & Times & (11.94) & (12.61) & (12.13) & $(12.4)$ \\
\hline & & & $100 \varepsilon_{r}$ & 0.15 & 0.15 & 0.15 & 0.15 \\
\hline & & \multirow[t]{4}{*}{2.0} & Exact & 213.02 & 211.3 & 593.46 & 591.74 \\
\hline & & & IE & 212.61 & 210.89 & 592.28 & 590.57 \\
\hline & & & Times & (12.16) & (12.58) & (11.92) & (12.29) \\
\hline & & & $100 \varepsilon_{r}$ & 0.19 & 0.19 & 0.2 & 0.2 \\
\hline & \multirow[t]{12}{*}{0.4} & \multirow[t]{4}{*}{1.0} & Exact & 98.48 & 96.77 & 269.43 & 267.71 \\
\hline & & & IE & 98.39 & 96.67 & 269.16 & 267.44 \\
\hline & & & Times & (12.23) & (12.23) & (12.26) & (12.53) \\
\hline & & & $100 \varepsilon_{r}$ & 0.1 & 0.1 & 0.1 & 0.1 \\
\hline & & \multirow[t]{4}{*}{1.5} & Exact & 160.78 & 159.06 & 442.62 & 440.9 \\
\hline & & & IE & 160.55 & 158.83 & 441.96 & 440.24 \\
\hline & & & Times & (12.25) & $(12.25)$ & $(12.26)$ & (12.37) \\
\hline & & & $100 \varepsilon_{r}$ & 0.15 & 0.15 & 0.15 & 0.15 \\
\hline & & \multirow[t]{4}{*}{2.0} & Exact & 262.04 & 260.32 & 726.71 & 724.99 \\
\hline & & & IE & 261.53 & 259.81 & 725.27 & 723.55 \\
\hline & & & Times & (12.36) & (12.56) & (12.23) & (12.26) \\
\hline & & & $100 \varepsilon_{r}$ & 0.2 & 0.19 & 0.2 & 0.2 \\
\hline & \multirow[t]{12}{*}{0.6} & \multirow[t]{4}{*}{1.0} & Exact & 120.51 & 118.79 & 329.3 & 327.58 \\
\hline & & & IE & 120.39 & 118.68 & 328.97 & 327.26 \\
\hline & & & Times & (12.23) & (12.49) & $(12.26)$ & $(12.46)$ \\
\hline & & & $100 \varepsilon_{r}$ & 0.1 & 0.1 & 0.1 & 0.1 \\
\hline & & \multirow[t]{4}{*}{1.5} & Exact & 197.1 & 195.38 & 541.33 & 539.61 \\
\hline & & & IE & 196.81 & 195.09 & 540.53 & 538.81 \\
\hline & & & Times & (12.22) & (12.57) & (12.29) & (12.54) \\
\hline & & & $100 \varepsilon_{r}$ & 0.15 & 0.15 & 0.15 & 0.15 \\
\hline & & \multirow[t]{4}{*}{2.0} & Exact & 321.91 & 320.19 & 889.46 & 887.74 \\
\hline & & & IE & 321.28 & 319.57 & 887.69 & 885.98 \\
\hline & & & Times & $(12.22)$ & $(12.46)$ & (12.23) & (12.47) \\
\hline & & & $100 \varepsilon_{r}$ & 0.2 & 0.2 & 0.2 & 0.2 \\
\hline
\end{tabular}

ical solution of the integral equation $j^{\mathrm{IE}}(x)$. Notice that there is a relative error less than $0.2 \%$ between the analytical expression and the Gauss-Legendre numerical scheme for integral equation (22) with $m=500$ nodes. The computational times for the exact formula are less than $1 \mathrm{~s}$ while the numerical integral equation times are approximately $11 \mathrm{~min}$.

The numerical results for the $\mathrm{MA}(2)$ case are shown in Table 2 . The results obtained from the two 
Table 3 Comparison of ARL(1) values for MA(1) process from explicit formula (exact) and numerical approximation (IE) for $\mathrm{ARL}_{0}=370, \theta=0.23, a=4, b=1.7$, and $m=500$.

\begin{tabular}{lccc}
\hline$\lambda$ & \multicolumn{2}{c}{$\theta=0.23$} & $100 \varepsilon_{r}$ \\
\cline { 2 - 3 } & Exact & IE & \\
\hline 1.0 & 371.323 & 370.701 & 0.168 \\
1.1 & 215.845 & 215.518 & 0.151 \\
1.2 & 137.285 & 137.097 & 0.137 \\
1.3 & 93.593 & 93.475 & 0.126 \\
1.4 & 67.389 & 67.312 & 0.115 \\
1.5 & 50.695 & 50.6407 & 0.106 \\
\hline
\end{tabular}

Table 4 Comparison of $\mathrm{ARL}_{1}$ values for MA(1) process from explicit formula (exact) and numerical approximation (IE) for $\mathrm{ARL}_{0}=500, \theta=0.23, a=4, b=2$, and $m=500$.

\begin{tabular}{lccc}
\hline$\lambda$ & \multicolumn{2}{c}{$\theta=0.23$} & $100 \varepsilon_{r}$ \\
\cline { 2 - 3 } & Exact & IE & \\
\hline 1.0 & 499.366 & 498.381 & 0.197 \\
1.1 & 281.652 & 282.154 & 0.178 \\
1.2 & 174.955 & 175.238 & 0.162 \\
1.3 & 116.898 & 117.071 & 0.148 \\
1.4 & 82.726 & 82.839 & 0.136 \\
1.5 & 61.305 & 61.381 & 0.125 \\
\hline
\end{tabular}

methods are again in good agreement with less than $0.2 \%$ relative errors for a range of parameter values. The computational times based on the exact solution take less than one second while the numerical integral equation takes approximately 12-13 min.

Tables 3 and 4 show a comparison of the exact and numerical solutions for an MA(1) process for given $\mathrm{ARL}_{0}=370$ and 500, respectively. In Table 3, we assume ARL $=370, a=4, b=1.7$, and $\theta=0.23$ and the number of division points in the GaussLegendre rule $m=500$. For $\lambda=1$ the process is in control whereas for $\lambda>1$ the process is out of control. The first row of Table 3 therefore shows values of $\mathrm{ARL}_{0}$ and rows 2-6 show values of $\mathrm{ARL}_{1}$. In Table 4, we assume ARL $=500, a=4, b=2$, and $\theta=0.23$ and the number of division points in the Gauss-Legendre rule $m=500$. As in Table 3, the first row shows the values of $\mathrm{ARL}_{0}$ and rows 2-6 show values of $A R L_{1}$

Tables 5-6 show a comparison of the exact and numerical schemes for an exponential second order moving average process MA(2) for $\mathrm{ARL}_{0}=370$ and 500 , respectively. Table 5 shows the results for $\mathrm{ARL}_{0}=370, \theta_{1}=0.65, \theta_{2}=0.24, a=4, b=1.3$.
Table 5 Comparison of ARL(1) values for MA(2) process from explicit formula (exact) and numerical approximation (IE) for $\mathrm{ARL}_{0}=370, \theta_{1}=0.65, \theta_{2}=0.24, a=4$, $b=1.3$, and $m=500$.

\begin{tabular}{|c|c|c|c|}
\hline \multirow[t]{2}{*}{$\lambda$} & \multicolumn{2}{|c|}{$\theta_{1}=0.65 \quad \theta_{2}=0.24$} & \multirow[t]{2}{*}{$100 \varepsilon_{r}$} \\
\hline & Exact & IE & \\
\hline 1.0 & 371.328 & 370.948 & 0.013 \\
\hline 1.1 & 216.580 & 216.545 & 0.093 \\
\hline 1.2 & 138.176 & 138.103 & 0.085 \\
\hline 1.3 & 94.456 & 94.383 & 0.077 \\
\hline 1.4 & 68.171 & 68.122 & 0.072 \\
\hline 1.5 & 51.385 & 51.351 & 0.066 \\
\hline
\end{tabular}

Table 6 Comparison of ARL(1) values for MA(2) process from explicit formula (exact) and numerical approximation (IE) for $\mathrm{ARL}_{0}=500, \theta_{1}=0.65, \theta_{2}=0.24, a=4$, $b=1.33$, and $m=500$.

\begin{tabular}{|c|c|c|c|}
\hline \multirow[t]{2}{*}{$\lambda$} & \multicolumn{2}{|c|}{$\theta_{1}=0.65 \quad \theta_{2}=0.24$} & \multirow[t]{2}{*}{$100 \varepsilon_{r}$} \\
\hline & Exact & IE & \\
\hline 1.0 & 500.455 & 499.795 & 0.132 \\
\hline 1.1 & 283.886 & 283.547 & 0.112 \\
\hline 1.2 & 176.948 & 176.755 & 0.109 \\
\hline 1.3 & 118.591 & 118.473 & 0.100 \\
\hline 1.4 & 84.147 & 84.069 & 0.093 \\
\hline 1.5 & 62.498 & 62.445 & 0.085 \\
\hline
\end{tabular}

For Table 6 the parameter values are $\mathrm{ARL}_{0}=500$, $\theta_{1}=0.65, \theta_{2}=0.24, a=4, b=1.33$. In both cases, there is good agreement between the exact and numerical results with differences of less than $0.1 \%$. Note that, as for the MA(1) results, $\lambda=1$ is assumed to be in-control parameter value and $\lambda>1$ to be out-of-control parameter values.

\section{CONCLUSIONS}

We have derived explicit expressions for the ARL of CUSUM charts for observations modelled as a moving average process of order $q(\operatorname{MA}(q))$ with exponential white noise. We have also used a GaussLegendre quadrature scheme to solve the integral equations for the ARL of CUSUM charts for MA(q) processes. We have shown by numerical computations that the explicit expression and the numerical scheme give results that are in very good agreement. We have shown that the explicit expression gives a very fast and effective method for calculating ARL for CUSUM charts with computation times of less than $1 \mathrm{~s}$ compared with computation times of approximately $12 \mathrm{~min}$ for the Gauss-Legendre scheme. 


\section{REFERENCES}

1. Page ES (1954) Continuous inspection schemes. Biometrika 41, 100-14.

2. Mazalov VV, Zhuravlev DN (2002) A method of cumulative sums in the problem of detection of traffic in computer networks. Program Comput Software 28, 342-8.

3. Hana D, Tsungb T, Lic Y, Xiana J (2010) Detection of changes in a random financial sequence with a stable distribution. J Appl Stat 37, 1089-111.

4. Corbett CJ, Pan JN (2002) Evaluating environmental performance using statistical process control techniques. Eur J Oper Res 139, 68-83.

5. Kennedy PD (1975) Some martingales related to cumulative sum test and single-server queues. Stoch Process Appl 4, 261-9.

6. Lim TO, Soraya A, Ding LM, Morad Z (2002) Assessing doctors' competence: application of CUSUM technique in monitoring doctors' performance. Int $J$ Qual Health Care 14, 251-8.

7. Noyez L (2009) Control charts, CUSUM techniques and funnel plots. A review of methods for monitoring performance in healthcare. Interact Cardiovasc Thorac Surg 9, 494-9.

8. Crowder SV (1978) A simple method for studying run length distributions of exponentially weighted moving average charts. Technometrics 29, 401-7.

9. Srivastava MS, Wu Y (1997) Evaluation of optimum weights and average run lengths in EWMA control schemes. Comm Stat Theor Meth 26, 1253-67.

10. Brook D, Evans DA (1972) An approach to the probability distribution of CUSUM run length. Biometrika 59, 539-48.

11. Lucas JM, Saccucci MS (1990) Exponentially weighted moving average control schemes: properties and enhancements. Technometrics 32, 1-29.

12. Zhonghua L, Changliang Z, Zhen G, Zhaojun W (2013) The computational of average run length and average time to signal: an overview. $J$ Stat Comput Simulat 84, 1779-802.

13. Sukparungsee S, Novikov AA (2008) Analytical approximations for detection of a change-point in case of light-tailed distributions. J Qual Meas Anal 4, 49-56.

14. Areepong Y (2009) An integral equation approach for analysis of control charts. PhD thesis, Univ of Technology, Australia.

15. Mititelu G, Areepong Y, Sukparungsee Novikov AA (2010) Explicit analytical solutions for the average run length of CUSUM and EWMA charts. East West $J$ Math 1, 253-65.

16. Petcharat K, Areepong Y, Sukparungsee S, Mititelu G (2011) Fitting Pareto distributions with hyperexponential to evaluate the average run length for cumulative sum chart. Int J Pure Appl Math 77, 233-44.

17. Yashchin M (1993) Performance of CUSUM control schemes for serially correlated observations. Technometrics 35, 37-52.

18. Jacob PA, Lewis PAW (1977) A mixed autoregressivemoving average exponential sequence and point process EARMA(1,1). Adv Appl Probab 1, 87-104.

19. Lawrance JA, Lewis PAW (1977) An exponential moving-average sequence and point process (EMA1). J Appl Probab 14, 98-113.

20. Mohamed I, Hocine F (2010) Bayesian estimation of an AR(1) process with exponential white noise. Statistics 37, 365-72.

21. Kirk WA, Khamsi MA (2001) An Introduction to Metric Spaces and Fixed Point Theory, Wiley, New York. 